\title{
Optical radiation propagation based on Green's functions in biological skin tissues for enhanced coherence contrast
}

Ganoza-Quintana, J. L., Fanjul-Vélez, F., Arce-Diego, J. L.

J. L. Ganoza-Quintana, F. Fanjul-Vélez, J. L. Arce-Diego, "Optical radiation propagation based on Green's functions in biological skin tissues for enhanced coherence contrast," Proc. SPIE 11238, Optical Interactions with Tissue and Cells XXXI, 1123806 (20 February 2020); doi:

$10.1117 / 12.2545008$

SPIE. Event: SPIE BiOS, 2020, San Francisco, California, United States 


\title{
Optical radiation propagation based on Green's functions in biological skin tissues for enhanced coherence contrast
}

\author{
J. L. Ganoza-Quintana, F. Fanjul-Vélez*, J. L. Arce-Diego* \\ Applied Optical Techniques Group, TEISA Department, University of Cantabria, Av de los Castros \\ s/n, 39005, Santander (Spain)
}

\begin{abstract}
Medical applications of treatment, diagnosis and surgery can greatly benefit from the use of optical radiation. Every biomedical optical technique depends strongly on light propagation. The spatial configuration and the characteristics of optical radiation at each spatial point greatly influence the outcome of the previously mentioned applications. Light properties as it traverses biological tissues are particularly relevant in optical diagnosis. Diagnosis by optical radiation is usually based on pure intensity measurements. Consequently, there is a general lack of enough contrast, as it is based on pure absorption and scattering differences. Enhanced contrast can be achieved by taking into account other light parameters, such as coherence or polarization. These parameters present a much more complex evolution, and are strongly dependent on the incident optical beam properties, as long as on the biological medium characteristics. The statistical nature of the process makes it convenient to use random beams and even random media in the models. These additional parameters could represent the possibility to distinguish malignant from healthy biological tissues, when intensity contrast is not enough. What is more, beam characteristics could be chosen in order to produce desired spatial distributions of radiation inside biological tissues, or to provide an adequate interpretation of diagnostic parameters. In this work, optical random beams, mainly Gaussian-based, are employed to model light propagation in turbid biological tissues by Green's functions. Coherence and spectral characteristics of the beam are considered. The model is applied to skin pathologies, such as basocellular or squamous cell carcinoma.
\end{abstract}

Keywords: propagation of light, optical properties, numerical approaches for light-tissue interactions, scattering in biological tissues, Green's functions in optics

\section{INTRODUCTION}

Medical applications of treatment, diagnosis and surgery can greatly benefit from the use of optical radiation. Biomedical optical techniques of treatment, characterization and surgery depend on the propagation of light in biological tissues [1]. The accuracy of light propagation and a priori estimates are particularly significant in diagnostic techniques. As biological tissues are turbid media, with great influence of scattering, it is necessary to adequately analyze its influence on optical propagation parameters, such as coherence. The influence of a scatterers distribution can be analyzed using Green's functions [2]. In this work, these functions are used for the analytical study of the complexity of the propagation of light as electromagnetic radiation in those turbid media that are biological tissues.

\section{THEORETICAL MODEL}

Scattering in biological media represents one of the most limiting effects for optical diagnostic imaging. Along with light intensity, coherence is particularly affected by the scattering mechanism [3]. The scattered wave $U_{s}(r, \omega)$ from a monochromatic incident wave $U_{i}(r, \omega)$ can be expr essed as a to ta $l U(r, \omega)$ wav e, a ssumin g the fir st-o rder Born approximation, as:

$$
U(r, \omega) \sim U_{i}(r, \omega)+\int_{D} F\left(r^{\prime}, \omega\right) U_{i}\left(r^{\prime}, \omega\right) G\left(\left|r-r^{\prime}\right|, \omega\right) d^{3} r^{\prime}
$$

In equation (1), $F(r, \omega)$ is the scattering potential of the medium, and $G\left(\left|r-r^{\prime}\right|, \omega\right)$ is the outgoing free-space Green function. If incident light is partially coherent, as it is a more general case, with a cross-spectral density function $W_{i}\left(r_{1}, r_{2}, \omega\right)$, the cross-spectral density of the scattered wave can be expressed as:

*fanjulf@unican.es; arcedj@unican.es; phone +34 94220 67 30; fax +34 9422018 73; www.teisa.unican.es/toa

Optical Interactions with Tissue and Cells XXXI, edited by Bennett L. Ibey, Norbert Linz, Proc. of SPIE

Vol. 11238, 1123806 · C 2020 SPIE · CCC code: 1605-7422/20/\$21 · doi: 10.1117/12.2545008 


$$
W_{s}\left(r_{1}, r_{2}, \omega\right)=\iint_{D} W_{i}\left(r_{1}^{\prime}, r_{2}^{\prime}, \omega\right) F^{*}\left(r_{1}^{\prime}, \omega\right) F\left(r_{2}^{\prime}, \omega\right) G^{*}\left(\left|r_{1}-r_{1}^{\prime}\right|, \omega\right) G\left(\left|r_{2}-r_{2}^{\prime}\right|, \omega\right) d^{3} r_{1}^{\prime} d^{3} r_{2}^{\prime}
$$

The cross-spectral density can be expressed as a function of the spectral densities $S_{i}\left(r_{1}^{\prime}, \omega\right)$ and $S_{i}\left(r_{2}^{\prime}, \omega\right)$, and the spectral degree of coherence $\mu_{i}\left(r_{1}^{\prime}, r_{2}^{\prime}, \omega\right)$ :

$$
W_{s}\left(r_{1}, r_{2}, \omega\right)=\iint_{D} \sqrt{S_{i}\left(r_{1}^{\prime}, \omega\right)} \sqrt{S_{i}\left(r_{2}^{\prime}, \omega\right)} \mu_{i}\left(r_{1}^{\prime}, r_{2}^{\prime}, \omega\right) F^{*}\left(r_{1}^{\prime}, \omega\right) F\left(r_{2}^{\prime}, \omega\right) G^{*}\left(\left|r_{1}-r_{1}^{\prime}\right|, \omega\right) G\left(\left|r_{2}-r_{2}^{\prime}\right|, \omega\right) d^{3} r_{1}^{\prime} d^{3} r_{2}^{\prime}
$$

The Gaussian Schell-model (GSM) is based on the cross-spectral density function, which has the structure [4],

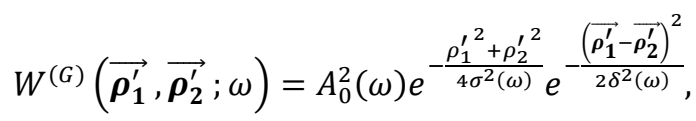

where superscript (G) stands for the Gaussian Schell-model, $A_{0}^{2}$ is the maximum value of the spectral density (attained on the axis) and the root-mean-square (r.m.s.) widths $\sigma^{2}$ and $\delta^{2}$ are independent of position but generally depend on frequency.

The optical turbulence is well explained by the presence of irregularities in the refractive index or, so-called, "turbulent eddies," appearing due to fluctuations in various physical properties of matter, such as temperature, pressure and concentration of inhomogeneous chemical content. Such eddies are created in different types of matter through certain physical/chemical/biological mechanisms. Shearing and mixing of different parts of the irregular structures under influence of winds in atmosphere, currents in the ocean, cell growth and fluid transfer in bio-tissues lead to a mechanism of energy transfer among eddies of different sizes. The largest possible size of an eddy in the turbulent process is taken as the definition of the outer scale $L_{0}$ of turbulence. Larger eddies break down further into smaller ones with energy until the size of the eddy reaches the lower limit when the energy dissipates. The size of the smallest eddy before dissipation defines the inner scale $l_{0}[5]$.

The solution of Helmholtz's equation can be found for the field $U(\vec{r}, \omega)$ in the form of the extended Huygens-Fresnel integral,

$$
U(\overrightarrow{\boldsymbol{r}}, \omega)=-\frac{i k e^{i k z}}{2 \pi z} \int U\left(\overrightarrow{\boldsymbol{\rho}^{\prime}}, \omega\right) e^{i k \frac{(\overrightarrow{\boldsymbol{\rho} \prime}-\overrightarrow{\boldsymbol{\rho}})^{2}}{2 z}} e^{\Psi\left(\overrightarrow{\boldsymbol{\rho}^{\prime}}, \overrightarrow{\boldsymbol{r}} ; \omega\right)} d^{2} \rho^{\prime}
$$

where $\Psi\left(\overrightarrow{\boldsymbol{\rho}^{\prime}}, \overrightarrow{\boldsymbol{r}} ; \omega\right)$ is the complex phase perturbation caused by the random distribution of the index of refraction in the medium.

A scalar GSM light beam is incident on a soft anisotropic biological tissue with anisotropy factors $\mu_{x}=\mu_{z} \neq \mu_{y}$ in the plane $z=0$, termed source plane or plane of incidence and propagates through it in the positive half-space $z>0$.

For biological tissues with geometrical anisotropy the three-dimensional power spectrum can be written in the following form, in similarity with other anisotropic random media,

$$
\Phi_{n}\left(\kappa_{x}, \kappa_{y}, 0\right)=\frac{(2 \pi)^{3} \sigma_{n}^{2} \mu_{x} \mu_{y} \mu_{z} e^{-\frac{\kappa_{x}^{2}}{\kappa_{m x}^{2}}-\frac{\kappa_{y}^{2}}{\kappa_{m y}^{2}}}}{\kappa_{0}^{3-\alpha}\left[\kappa_{0}^{2}+4 \pi^{2}\left(\mu_{x}^{2} \kappa_{x}^{2}+\mu_{y}^{2} \kappa_{y}^{2}\right)\right]^{\frac{\alpha}{2}}}, \quad \begin{aligned}
& \mathbf{3}<\mathbf{4} .
\end{aligned}
$$

Where $\alpha$ is the power spectrum slope, $\sigma_{n}^{2}$ is the variance of the refractive index of the bio-tissue, $\mu_{x}, \mu_{y}$ and $\mu_{z}$ are the anisotropic strength coefficients in each direction. Further, $\overrightarrow{\boldsymbol{\kappa}_{\mathbf{0}}}$ is the large-scale cut-off frequency vector with magnitude $\kappa_{0}=\sqrt{\kappa_{x 0}^{2}+\kappa_{y 0}^{2}+\kappa_{z 0}^{2}}$ and components $\kappa_{x 0}=\frac{2 \pi}{L_{x}}, \kappa_{y 0}=\frac{2 \pi}{L_{y}}, \kappa_{z 0}=\frac{2 \pi}{L_{z}}$, with $L_{x}, L_{y}$ and $L_{z}$ being the outer scales along $x, y$ and $z$, directions, respectively: $L_{x}=\mu_{x} L_{0}, L_{y}=\mu_{y} L_{0}$ and $L_{z}=\mu_{z} L_{0}$. Small-scale cut-off frequency vector $\overrightarrow{\boldsymbol{\kappa}_{\boldsymbol{m}}}$ has magnitude $\kappa_{m}=\sqrt{\kappa_{x m}^{2}+\kappa_{y m}^{2}+\kappa_{z m}^{2}}$ and components $\kappa_{x m}=\frac{2 \pi}{l_{x}}, \kappa_{y m}=\frac{2 \pi}{l_{y}}, \kappa_{z m}=$ $\frac{2 \pi}{l_{z}}$ with $l_{x}=\mu_{x} l_{0}, l_{y}=\mu_{y} l_{0}$ and $l_{z}=\mu_{z} l_{0}$. 
For an anisotropic turbulent medium and the vector with Cartesian coordinates $\overrightarrow{\boldsymbol{\rho}}=(\xi, \eta)$, the spectral density is given by,

$$
S(\overrightarrow{\boldsymbol{\rho}}, z)=\frac{1}{\sqrt{\Delta_{x}(z) \Delta_{y}(z)}} e^{-\frac{\xi^{2}}{2 \sigma_{0}^{2} \Delta_{x}(z)}} e^{-\frac{\eta^{2}}{2 \sigma_{0}^{2} \Delta_{y}(z)}}
$$

\section{RESULTS AND DISCUSSION}

For all the following simulations it has been used as a reference [6]. For all the examples below the numerical values of parameters are set to: $\lambda=0.6328 \mu \mathrm{m}, \sigma_{0}=3 \mathrm{~mm}, \sigma_{n}^{2}=4 \times 10^{-4}, L_{0}=5 \mu \mathrm{m}, l_{0}=0.2 \mu \mathrm{m}, \mu_{x}=1, \mu_{y}=$ $3, \mu_{z}=1, \alpha=3.5$, unless different values are specified in figure. In Figure 1 the power spectrum of a typical anisotropic biological tissue is plotted from Eq. (6) along the $\mathrm{x}$ - and $\mathrm{y}$ - directions. The discrepancy in the power spectrum along the two orthogonal directions is clearly seen. Figure 2 is plotted from Eq. (7) and it shows the changes in the spectral density of the beam along the propagation path, showing that the beam spreads much sooner in the path along the axis with the smaller anisotropic factor (y-axis) than that with the larger one (x-axis). As the propagation distance increases to values on the order of $1 \mathrm{~cm}$, the ratio of the semi-minor axis and the semi-major axis of the ellipse approaches $1 / 3$ being exactly the ratio of the anisotropic factors, $\mu_{x} / \mu_{y}$.

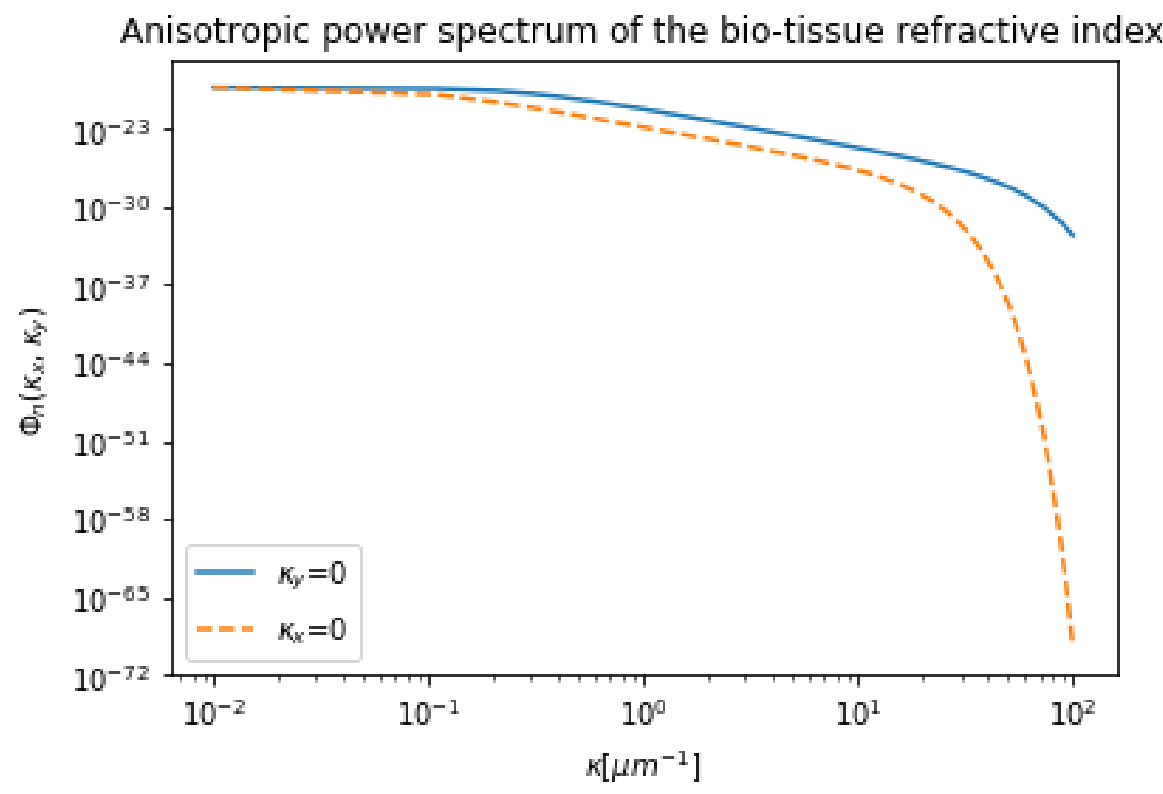

Figure 1. Anisotropic power spectrum of the bio-tissue refractive index along the $\mathrm{x}$ - and $\mathrm{y}$ - directions. 
Spectral density at $\mathrm{z}=1 \mathrm{~cm}$ with different anisotropy ratios $\mu_{x} / \mu_{y}$
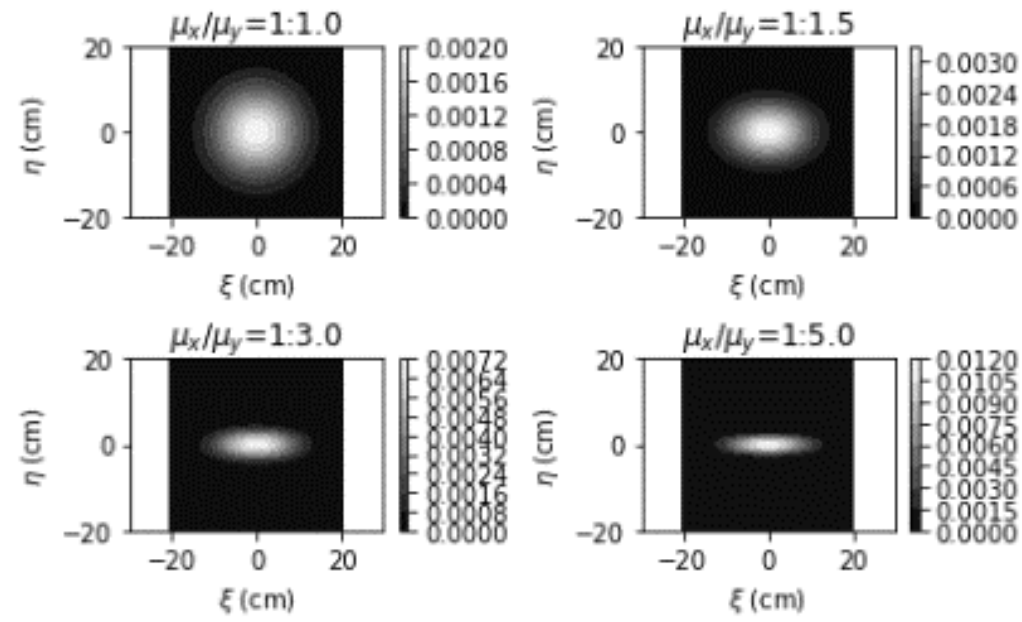

Figure 2. Spectral density of the coherent GSM beam propagating in the bio-tissue at $\mathrm{z}=1 \mathrm{~cm}$ with different anisotropy ratios.

\section{CONCLUSIONS}

Biomedical optical techniques of treatment, characterization and surgery are strongly dependent on light propagation. Further parameters based on polarization or coherence can provide increased contrast in diagnostic techniques. Light propagation in static highly scattering biological tissues can be analyzed by Green's functions, including coherence phenomena. The extended Huygens-Fresnel method has been applied for the analytical evaluation of the spectral density of the propagation beam and its coherence state, within the validity of the Markov approach. A variety of numerical examples have been provided illustrating the effects of the source and tissue parameters on the evolution of the beam. The results of this study may be useful in the medical diagnosis and treatment of anisotropic bio-tissues by optical radiation.

\section{ACKNOWLEDGEMENTS}

This work has been partially supported by the project "High-pressure driven plasmonic and luminescence properties of naked and core/shell metal-oxide nanocomposites" (PGC2018-101464-B-I00) of the Spanish Ministry of Science, Research and Universities, cofunded by FEDER funds, and by the San Cándido Foundation.

\section{REFERENCES}

[1] Girkin, J. M. and Carvalho, M. T., “The light-sheet microscopy revolution,” J. Opt. 20, 053002 (2018).

[2] Ganoza-Quintana, J. L., Fanjul-Vélez, F. and Arce-Diego, J. L., "Optical coherence propagation in biological tissues with significant scattering by Green’s functions," Optical Society of America, Frontiers in Optics, paper JTu3A.106 (2018).

[3] Ganoza-Quintana, J. L., Fanjul-Vélez, F., and Arce-Diego, J. L., "Light propagation in highly scattering biological tissues analyzed by Green's functions", Proc. SPIE 10876, Optical Interactions with Tissue and Cells XXX, 108760Q (2019).

[4] Korotkova O., [Random Light Beams: Theory and Applications], CRC Press, (2013).

[5] Andrews, L. C. and Phillips, R. L., [Laser beam propagation through random media], SPIE Press, (2005).

[6] Chen, X. and Korotkova, O., "Optical beam propagation in soft anisotropic biological tissues", OSA Continuum 1(3), 1055-1067 (2018). 